\title{
A MODEL FOR EVALUATING THE RISK EFFECTS ON CONSTRUCTION PROJECT ACTIVITIES
}

\author{
Usama Hamed ISSA ${ }^{1,2}$, Salah Attia MOSAAD ${ }^{3,4}$, Mohamed Salah HASSAN ${ }^{4}$ \\ ${ }^{1}$ Department of Civil Engineering, College of Engineering, Taif University, Taif, Saudi Arabia \\ ${ }^{2}$ Department of Civil Engineering, Faculty of Engineering, Minia University, Minia, Egypt \\ ${ }^{3}$ Project Management Division, Jabal Omar Development Company, Makkah, Saudi Arabia \\ ${ }^{4}$ Department of Mechanical Power and Energy, Faculty of Engineering, Minia University, Minia, Egypt
}

Received 06 February 2019; accepted 29 April 2019

\begin{abstract}
Cost overruns and time delays are considered to be very important challenges for the majority of construction projects. These challenges are typically attributed to their associated risks. Due to the risky and uncertain nature of construction projects, an increasing amount of attention is given to estimating and overcoming cost overruns and time delays. New techniques are being developed to help project managers to contractually complete projects within cost and time constraints. The objective of this study was to develop a new qualitative and quantitative risk analysis model that can be employed for construction projects. The proposed model, which is based on a fuzzy logic tool, consists of two modules for assessing risk factors that affect the main construction activities and computing the expected cost overruns and time delays that are associated with these risks. Using numerous logical rules, the model applies the probability of occurrences and impacts of the risks on the cost and time of the main activities. The Spearman and Kendall correlation coefficient tests are applied to verify and select a suitable membership function. Using four proposed membership functions, the results of these tests confirmed that the triangle membership function is suitable for the model. The model is verified by application to HVAC system activities in two actual construction projects, which serve as case studies. Two different methods are proposed and applied to quantify the cost overruns and time delays. The first method is based on determining the cost overruns and time delay values for each activity according to their weight in the system. Triple premise rules are proposed and applied in the second method, which is established to relate all activities. The results from the second method are more accurate compared with the first method based on actual data from the case study projects. In addition, the results demonstrated that the proposed model can be used to quantify the expected cost overrun and time delays in construction project activities and can be generalized and implemented in different construction activities.
\end{abstract}

Keywords: construction project activities, evaluating risks, cost overruns, time delays, risk analysis.

\section{Introduction}

The construction industry is considered to be one of the most dynamic and risky sectors in all countries. Many construction projects do not attain their desired goals due to the presence of risks and uncertainties intrinsic in the projects. Uncertainty exists in the success of large-scale construction projects in terms of cost, time consumed by technological constraints, large numbers of stakeholders, long duration, numerous capital requirements, and improperly defined project scope (Gohar, Khanzadi, \& Farmani, 2012). A major problem that occurs due to risks in most construction projects is the cost overruns or time delays that are incurred by a project, which cause an increase in the project cost or duration. Due to the undocumented data and particular behaviors of the construction projects, a probabilistic approach cannot usually be applied to quantify risks (Khazaeni, Khanzadi, \& Afshar, 2012). The majority of previous research has investigated the effect of risk on the cost overruns and time delays of construction projects in general, whereas few studies have explored the impact of these risks on the construction project activities. Consequently, researchers give considerable attention to overcome these problems in the future by the development of theoretical models to evaluate the cost overruns and time delays resulted from risks affecting construction activities.

${ }^{\star}$ Corresponding author. E-mail: sazaher@jodc.com.sa 
Risks in construction projects can be represented by risk factors that affect many project objectives. The most critical risk factors can be distinguished as capturing more attention (Rezakhani, 2012). Many risk factors that affect time and cost objectives are identified for many case studies (Rezakhani, 2011; Zhang \& Zou, 2007; Singh \& Trivedi, 2012; Senouci, Ismail, \& Eldin, 2016; Abd El Khalek, Aziz, \& Kamel, 2017; Nawar, Hosny, \& Nassar, 2017; Issa \& Ahmed, 2014; Issa, Farag, Abdelhafez, \& Ahmed, 2015). The risk assessment process requires an assessment of the probability or likelihood of the risk and impact (Zhang \& Zou, 2007). The assessment of the level of risk is a complex subject that is shrouded in uncertainty and vagueness (Rezakhani, 2012). Evaluating and analyzing the risks of a project and planning their management are the most critical steps in the project definition stage (Gohar et al., 2012). Risk management aims to identify the sources of risk and uncertainty, determine their impact, and develop appropriate management response (Rezakhani, 2011). The majority of risk analysis tools are based on statistical decision theory. However, contractors seldom use these tools in their analysis. In addition, an accurate risk assessment approach can be achieved by collecting information from previous experiences, probabilities, brainstorming sessions and individual knowledge. Fuzzy set theory is an important tool that can be employed to address uncertainties that are not statistical in nature (Issa, 2012a). More details concern risk analysis and fuzzy set theory will be introduced in the following sections.

The main purpose of this study was to propose and develop a new model that addresses cost overruns and time delays attributed to risks that affect construction project main activities. A suitable membership function will be selected and employed in the model. The proposed model consists of two modules. The first module, namely, Fuzzy Module for Qualitative Risk Analysis (FMQRA), can assess the factors priorities that produce cost overruns and time delays in construction projects. The second module, namely, Fuzzy Module for Cost overruns and Time delays Quantification (FMCTQ), determines the expected cost overruns and time delays for activities using two different methods. The risks commonly identified in Heating, Ventilation, and Air Conditioning (HVAC) system activities are used as a case study for applying the model. The model has been verified using data from two real projects in Saudi Arabia. In addition to the introduction and research methodology, the outlines of this paper include a review for risk analysis and fuzzy logic, proposed Fuzzy Risk Analysis Model development, Model Verification, Model limitations and Conclusions.

\section{Risk analysis}

Before managing the risks, they should be qualitatively and quantitatively analyzed. The majority of real-world risk analysis problems contain a mixture of quantitative and qualitative data (Morote \& Vila, 2011). Qualitative analysis relies on the subjective judgment of competent personnel to determine the total risk (Morote \& Vila, 2011). The identified risks can be qualitatively assessed to determine both the probability and the potential effect on project objectives, which enables the risks to be prioritized for further attention (Issa, 2012b). On the other hand, quantitative risk analysis in project management is the process of converting the impact of risk on a project into numerical terms. This numerical information is frequently used to determine the cost and time contingencies of a project (PM Institute, 2004). Many studies have explored and qualitatively assessed the risks; however, limited research has been dedicated to the quantitative assessment of the risky conditions/factors of construction projects (Zhang \& Zou, 2007).

Linguistic variables are considered the best way to represent the risks; however, it is difficult to analyze them numerically. Fuzzy logic can handle imprecise data sets including information featuring non-statistical uncertainties. Besides, the main advantage of fuzzy set theory compared to other methods is the ability to operate with linguistic variables (Cheng, Xu, \& Chen, 2018; Issa, Miky, \& Abdel-Malak, 2019). For these reasons, fuzzy sets can be used to qualify and quantify the linguistic variables which represent likelihood and severity (Abd El Khalek et al., 2017; Carr \& Tah, 2000). Recent researches utilized fuzzy logic theory in qualifying or quantifying data in construction projects. For example, fuzzy reasoning techniques provide a systematic tool to address quantitative data in the construction process (Zeng, An, \& Smith, 2007). Another quantitative method that is based on the fuzzy analytic hierarchy process approach has been provided to manage the risk of construction projects in an uncertain environment (Gohar et al., 2012). A quantitative model for the risk allocation process has been provided to support decision making in risk management in a manner that addresses the concerns of inappropriate risk allocation (Khazaeni et al., 2012). Quantitative analysis is based on a simultaneous evaluation of the impact of all identified and quantified risks (Ahmed, Issa, Farag, \& Abdelhafez, 2013). A risk assessment model is proposed based on the concepts of fuzzy set theory to evaluate the risk events during tunnel construction operations (Yazdani-Chamzini, 2014). A three-stage approach based on the fuzzy inference system under the environment of the Elena guideline is proposed to cope with the risky projects (Asadi, Zeidi, Mojibi, Yazdani-Chamzini, \& Tamosaitiene, 2018).

\section{Research methodology}

From previous research conducted by the authors (Mosaad, Issa, \& Hassan, 2018), the main activities of the HVAC systems in the construction project and the risk factors that affect each activity have been identified. Three activities associated with the installation of HVAC systems were declared as follows: $(A)$ air distribution duct work; $(B)$ chilled water distribution pipe work; and (C) HVAC equipment installation. Fifty-five risk factors that affect each specific activity and that are common to all activities 
RESEARCH METHODOLOGY

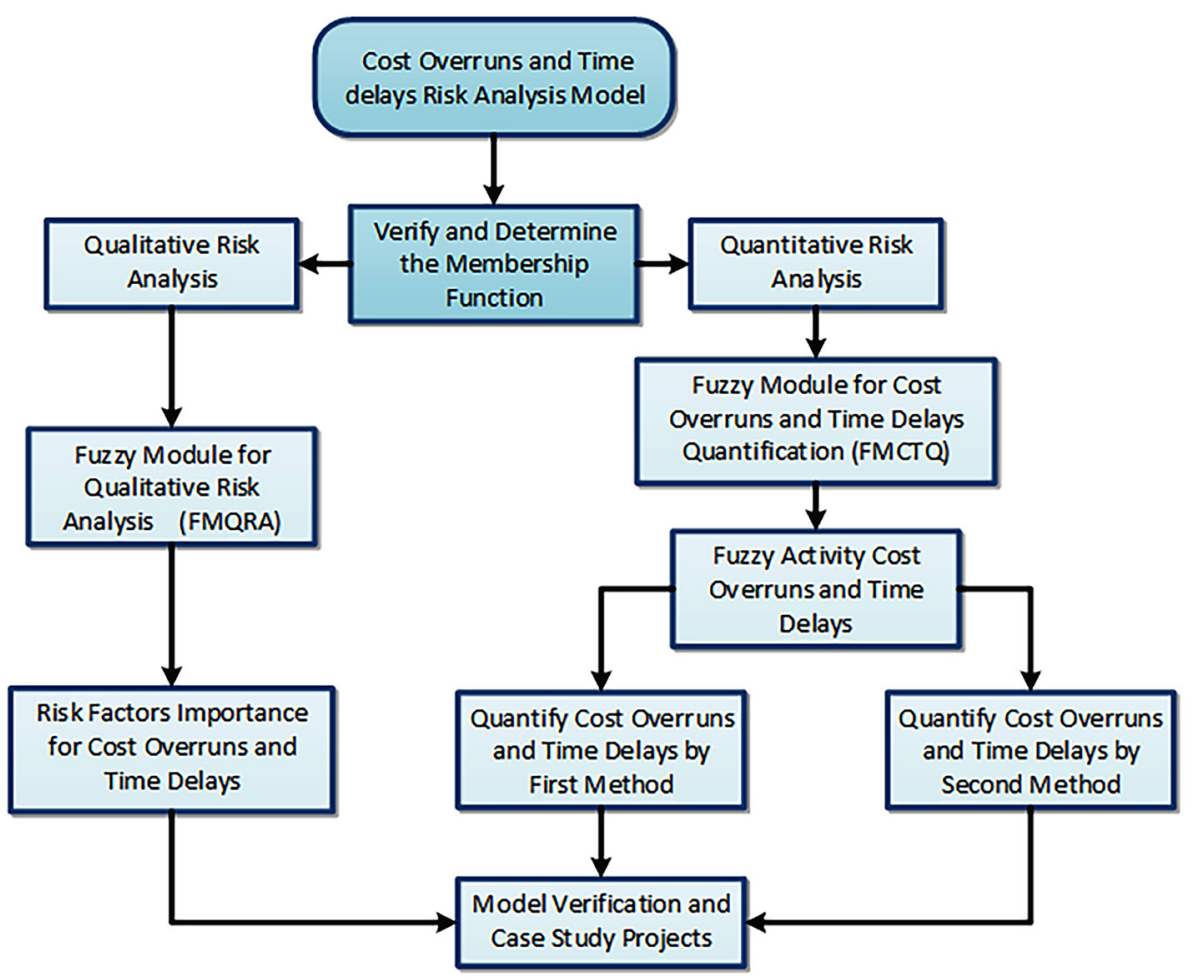

Figure 1. Summary of the research methodology

were also identified and categorized into four groups. The research methodology includes ranking and assessing the identified risk factors due to their importance using the first module FMQRA, which depends on combining the probability and impacts on cost or time for each risk factor. In addition, the cost overruns and time delays values are determined by the second module FMCTQ, which is based on determining fuzzy activity cost overruns or time delays for each activity using two methods. The first method is based on summing the cost overruns or time delays for each activity according to their weights in the system. A triple premise rule is proposed and applied in the second method and based on combining cost overruns or time delays values for all activities. Figure 1 shows a summary of the research methodology. Details about each module will be presented in the following sections. Finally, the model output data have been verified by applying two cases in country of Saudi Arabia.

\section{Proposed fuzzy risk analysis model}

The proposed new risk analysis model is developed by creating two different modules that address the main activities of construction projects and risks that affect them based on a fuzzy logic approach. The first module -FMQRA - is developed to assess risk factors in an acceptable and easy manner. FMCTQ - the second module - is developed to quantify the cost overruns and time delays that are based on many relationships concerning the impacts of risk fac- tors on the time and cost of activities by weighting many logical rules. Note that the model is wide-ranging and can be applied to all activities of construction projects after minor changes are implemented. The model will be compared to available data from the statistical analysis and real construction projects.

\subsection{Development of fuzzy module for qualitative risk analysis (FMQRA)}

FMQRA can be developed via three steps, namely, constructing the fuzzy membership function, specifying the fuzzy rule base and determining the fuzzy inference mechanism by using the fuzzy toolbox of MATLAB. The crisp inputs that will be used in this module comprise three indices: probability index $(P I)$, impact index for cost (IIC) and impact index for time (IIT). To assess the risks associated with activities in the construction projects, new risk indices represent the output of this module, namely, Fuzzy Risk Index for Cost (FRIC) and Fuzzy Risk Index for Time (FRIT). The FRIC indicates the importance or the magnitude of a risk factor based on a relationship between its probability of occurrence and its impact on cost to assess the expected cost overruns. The FRIT represents the importance or the magnitude of a risk factor based on the relationship between its probability of occurrence and its impact on time to assess the expected time delays (see Figure 2). 


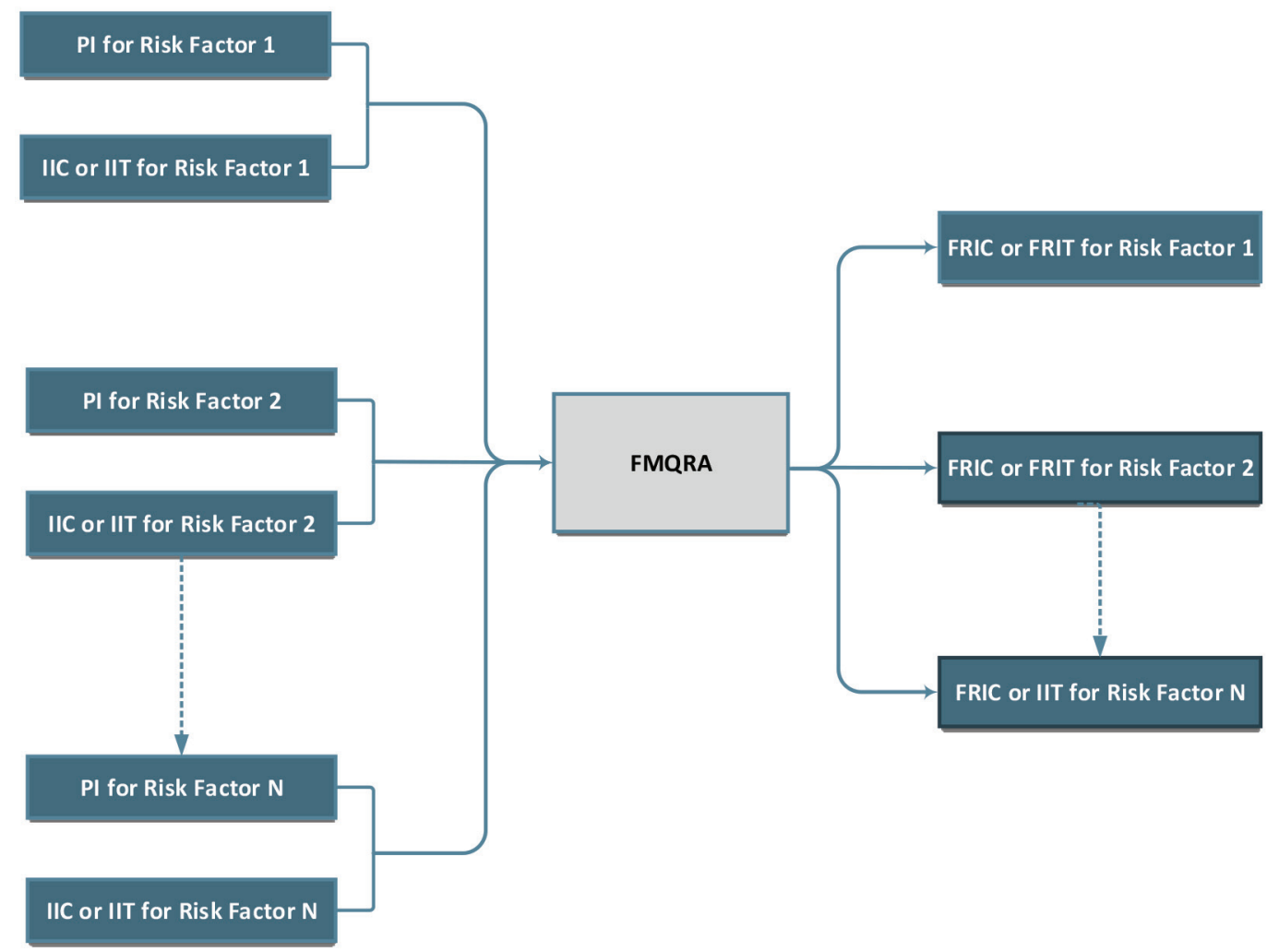

Figure 2. Fuzzy module for qualitative risk analysis (FMQRA)

\subsubsection{Aggregation and defuzzification process for FMQRA}

The aggregation rules in this module follow the commonsense behavior of the system and are written in terms of membership function linguistic labels. The risk magnitude can usually be assessed by considering two fundamental risk parameters - risk likelihood and risk severity - as stated in Issa and Ahmed (2014). The relationship in this module (two inputs and one output system) are needed to introduce logical rules for the two inputs (probability of occurrence and impact for each risk factor), which are considered the only available data. Rules, which connect input variables to output variables, are defined to perform inference. Each rule is a logical inference and depends on the state of the input and output variables. With the help of fuzzy rules, values can be incorporated between the conventional evaluation of the precise logic 1 and 0 . Logical operations such as "and", "or", "not" and "if-then" are included. The "(AND) only..." form is employed in this study to relate the input variable to the output variables in terms of the linguistic variables. The number of rules depends on the number of inputs and outputs and the required performance of the system. The Mamdanitype fuzzy inference method is employed in this study due to its extensive application in the construction industry (Sharma \& Goyal, 2014). Let there exist a relationship between the two input probabilities of occurrence for a certain risk factor represented by its probability index $(P I)$, the impact of the same risk factor on a project objective represented by its impact index (II), and the output of the model, which is the importance of the risk factor represented by the fuzzy risk index (FRI). This relation can be represented by a double premise rule, as follows:

- If the probability of occurrence and impact on cost, then cost overruns;

- If the probability of occurrence and impact on time, then time delays.

The inputs (probability of occurrence, the impact on cost and the impact on time) can be represented by PI, IIC, and IIT, respectively, whereas the outputs (cost overruns and time delays) can be represented by FRIC and FRIT, respectively.

Mathematically, the double premise rule can be transformed to the following rules:

- If $(P I)$ and (IIC), then (FRIC);

- If (PI) and (IIT), then (FRIT).

The linguistic description assigned to a fuzzy set in this module is similar to the labels used in the field survey [19]. For example, for the input factor, the probability of occurrence for a certain risk factor - the fuzzy label - can be described as follows: very low (VL), low (L), medium (M), high $(\mathrm{H})$, or very high $(\mathrm{VH})$. Each label is associated with a fuzzy set, as shown in Table 1.

Many relationships with varying values of $P I, I I$, and FRI exist. These relationships can be represented using Fuzzy Associative Memories (FAMs) using the method suggested by Kosko (1992), Carr and Tah (2000, 2001), Issa (2012a, 2012b). The interrelationships among the FAMs are similar those introduced by Carr and Tah (2001). 
Table 1. FAMs rules for calculating the output of FMQRA

\begin{tabular}{|c|c|c|c|c|c|c|}
\hline \multirow{2}{*}{\multicolumn{2}{|c|}{ Risk Scale }} & \multicolumn{5}{|c|}{ Risk Impact Index (II) } \\
\hline & & \multirow{2}{*}{$\frac{V L}{V L}$} & \multirow{2}{*}{$\frac{\mathrm{L}}{\mathrm{VL}}$} & \multirow{2}{*}{$\frac{\mathrm{M}}{\mathrm{L}}$} & \multirow{2}{*}{$\frac{\mathrm{H}}{\mathrm{L}}$} & \multirow{2}{*}{$\frac{\mathrm{VH}}{\mathrm{M}}$} \\
\hline \multirow{5}{*}{ 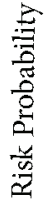 } & VL & & & & & \\
\hline & $\mathrm{L}$ & $\mathrm{VL}$ & $\mathrm{L}$ & $\mathrm{L}$ & $\mathrm{M}$ & $\mathrm{M}$ \\
\hline & $\mathrm{M}$ & $\mathrm{L}$ & $\mathrm{L}$ & $\mathrm{M}$ & $\mathrm{M}$ & $\mathrm{H}$ \\
\hline & $\mathrm{H}$ & $\mathrm{L}$ & $\mathrm{M}$ & $\mathrm{M}$ & $\mathrm{H}$ & $\mathrm{VH}$ \\
\hline & $\mathrm{VH}$ & $\mathrm{M}$ & $\mathrm{M}$ & $\overline{\mathrm{H}}$ & $\mathrm{VH}$ & VH \\
\hline
\end{tabular}

The process for determining the result or rule strength of the rule is performed by taking the minimum fuzzy input of antecedent 1 AND antecedent 2 , with minimum inferencing. This minimum result is equal to the consequent rule strength. If any consequents are equivalent, then the maximum rule strength between similar consequents, which is referred to as maximum or maximum inferencing, is employed; thus, minimum/maximum inferencing. This finding infers that the rule that is most true is taken. These rule strength values are referred to as fuzzy outputs.

$$
\begin{aligned}
& F R I C=P I \wedge I I C ; \\
& F R I T=P I \wedge I I T,
\end{aligned}
$$

where $\wedge$ refers to the intersection between the two inputs.

A sample of the rules extracted from the FAMs matrix are listed as follows:

- If the PI is low and the IIC is high, then the FRIC is medium;

- If the PI is high and the IIC is medium, then the FRIC is medium;

- If the PI is very low and the IIT is high, then the FRIT is low;

- If the PI is very high and the IIT is high, then the FRIT is very high.

All rules in this model have a weight equal to 1 .

Once the probability of occurrence and the impact of an individual risk factor are identified, their importance can be assessed using the fuzzy risk index, which can be computed using the proposed model.

\subsubsection{FMQRA application}

Three available indices will be used as inputs for this module, as calculated from the statistical analysis for every risk factor: PI, IIC, and IIT. The module outputs are the FRIC and the FRIT, which can be used to assess the risk factors. The FRIC represents the importance of the risk factor due to its probability of occurrence and its impact on the cost of a project, whereas the FRIT measures the importance of the risk factor due to its probability of occurrence and its impact on the time of the project. These two indices will be used to assess the risk factors by ranking them and illustrating a linear indication. To evaluate the results of this module, fifty-five risk factors for HVAC system activities that were previously identified (Mosaad et al., 2018) can be ranked due to their severity, which can be calculated as the magnitude of the probability of occurrence multiplied by the impact of the risk factor.

The risk factor index for cost $(R F I C)$ and the risk factor index for time (RFIT) were calculated using the following equations (Mosaad et al., 2018):

$$
\begin{aligned}
& R F I C=P I^{\star} I I C ; \\
& \text { RFIT }=P I^{\star} I I T .
\end{aligned}
$$

\subsubsection{Membership functions}

The definition of the membership function (MF) in fuzzy logic is a curve that defines how each point in the input space is mapped to a membership value (or degree of membership) between 0 and 1 . The simplest membership functions are formed using straight lines. The simplest membership function is a triangular-shaped membership function that has the function name trimf. This function consists of a collection of three points that form a triangle. The trapezoidal-shaped membership function trapmf has a flat top and is a truncated triangle curve. These straightline membership functions have the advantage of simplicity. Two membership functions are built on the Gaussian distribution curve: a simple Gaussian curve is gaussmf. The generalized bell membership function is specified by three parameters and has the function name gbellmf. The bell membership function has one more parameter than the Gaussian membership function; thus, it can approach a nonfuzzy set if the free parameter is tuned. Because of their smoothness and concise notation, Gaussian and bell membership functions are popular methods for specifying fuzzy sets. Both curves have the advantage of being smooth and nonzero at all points. Most previous research was distributed by triangular fuzzy numbers; however, the various membership functions need to be estimated to be as realistic as possible (Rezakhani, 2011). A triangleshaped membership function was employed by Carr and Tah (2001) in their risk assessment model using the cause and effect diagrams and utilized by Dikmen, Birgonul and Han (2007) to rate the cost overruns risk in international construction projects. The function was employed by Issa and Ahmed (2014) to assess the factors that control the quality of the driven pile activities. A trapezoid-shaped membership function was used by Zeng et al. (2007) to determine the risk magnitude. Rezakhani (2012) also applied the trapezoid-shaped function with the triangle- 
shaped function to describe the weights of the decision makers. A generalized bell-shaped membership function was used by Abd El Khalek, Aziz, and Kamel (2016) to evaluate the risk factors impact. A Gaussian-shaped membership function did not previously apply in the risk assessment models. In this research, four-member-shaped functions will be applied to select the best shaped function after comparison with the previous results from Mosaad et al. (2018), by determining the Spearman's rank correlation coefficient from the SPSS program. A fuzzy inference mechanism is a process of mapping a given input to an output using the theory of fuzzy sets. The Mamdani method was employed in this model since it has been widely accepted in fuzzy expert system development (Idrus, $\mathrm{Nu}$ ruddin, \& Rohman, 2011). As shown in Figure 3, the triangle-, trapezoid-, generalized bell- and Gaussian-shaped membership functions are applied for the comparison to select the best membership function shape. The linguistic description assigned to a fuzzy set in this model is similar to the labels used in the field survey (Mosaad et al., 2018). For example, for the input factor, the probability of occurrence for a certain risk factor - the fuzzy label - can be VL, L, M, H, or VH. Each label is associated with a fuzzy set.

\subsubsection{Selection MF using agreement tests}

The Spearman and Kendall correlation coefficient tests are two well-known measurements of nonparametric rank correlations that are applied to determine the strength of a link between any two sets of data (Issa \& Salama, 2018). SPSS software was used to calculate both correlation coefficients. Both correlation coefficients vary from -1 to +1 . The Spearman and Kendall tests were applied for the output from FMQRA using triangular, trapezoidal, generalized bell-shaped and Gaussian-shaped membership functions with the risk factors index. As shown in Figures 4(a), 4(b), the triangular, trapezoidal and Gaussianshaped membership function values of the Spearman and Kendall rank correlation coefficient $(\mathrm{R})$ are positive. Referring to Figures 4(a), 4(b), the strongest relationship was observed for the triangle-shaped membership function, with coefficient values of 0.65 and 0.7 for the Spearman test and 0.49 and 0.51 for the Kendall test in the case of the cost impact and time impact, respectively. The weakest relationship was observed for the generalized bell membership function with coefficient values of 0.35 and 0.43 for the Spearman test and 0.25 and 0.32 for the Kendall test in the case of the cost impact and time impact, respectively; however, it remains positive. A high degree of agreement between FRIC and FRIT is observed for the triangle-shaped membership function with RFIC and RFIT. The triangle-shaped membership function is selected in the proposed new risk analysis model.

\subsubsection{Results and discussion due to applying the FMQRA}

Once identifying and classifying the risk factors, the FMQRA determines which risk factors would potentially
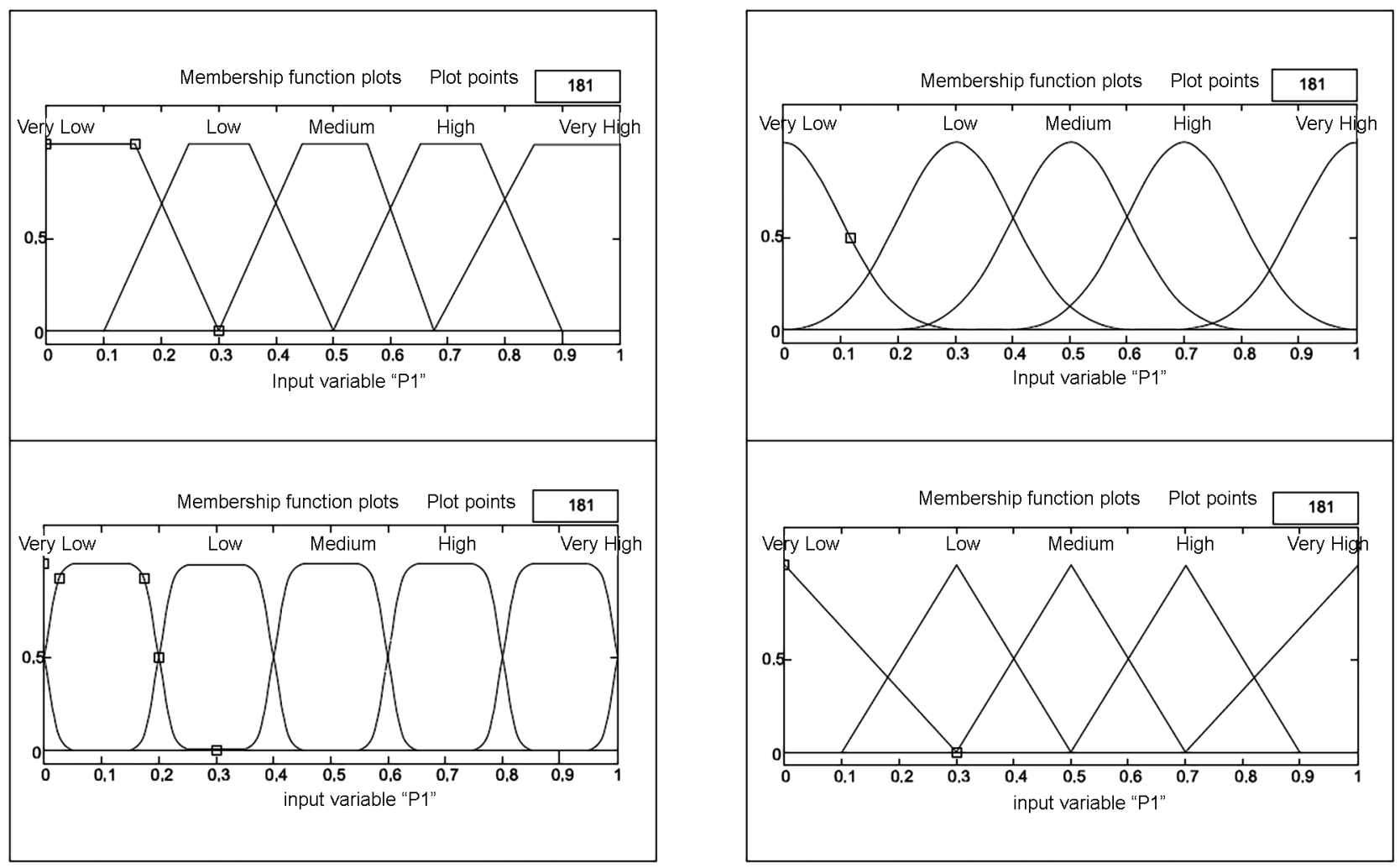

Figure 3. Membership functions in the proposed model 


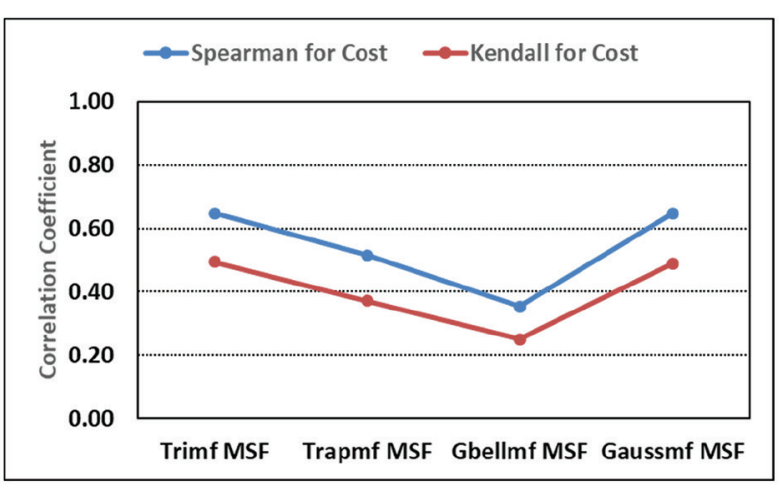

(a)

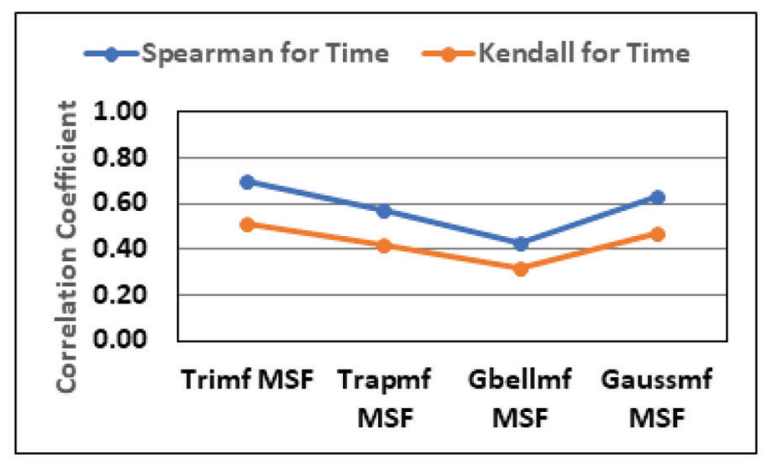

(b)

Figure 4. (a) Spearman and Kendall correlation coefficients for cost; (b) Spearman and Kendall correlation coefficients for time

have greater impacts on cost overruns and time delays. Figure 5 shows a comparison for ranking the risk factors based on the severity index for cost and the fuzzy risk index for cost, as calculated by the FMQRA. Figure 6 shows a comparison for ranking the risk factors based on the severity index for time and the fuzzy risk index for time. Based on the fuzzy risk indices for cost overruns and time delays for every risk factor, as calculated in Figures 5 and 6 , the risk factors importance can be compared using ranking tables for all risk factors. These results can be introduced for all professionals involved in construction projects. These results provide a useful reference for decision makers and investors who need to consider the major risks in HVAC systems.

Based on the results shown in Figures 5 and 6, the following conclusions can be obtained:

1. The most critical risk factor expected to cause the cost overruns and time delays of the HVAC system is Poor selection of valves that might cause more damage.

2. Many other risk factors (Wrong selections that might cause future rectification, Poor-quality shop and coordination drawings, Lack of specialized laborers, Equipment sizes clash with provided spaces, and Selecting fittings that might not be compatible with the pipe thickness) are considered to be critical and comprise the first ten risk factors that cause cost overruns and time delays.

3. The importance of the majority of the risk factors is similar in both the cost overruns and the time delays of the HVAC system.

\subsection{Development of fuzzy module for cost overruns and time delays quantification (FMCTQ)}

A new approach provides a module to determine the expected quantification of cost overruns and time delays in construction projects. The first step of developing the module is defining the inputs and the outputs of the module. The inputs are the top 10 risk factors that affect the cost and time impact for each activity $(A, B$ and $C)$, and

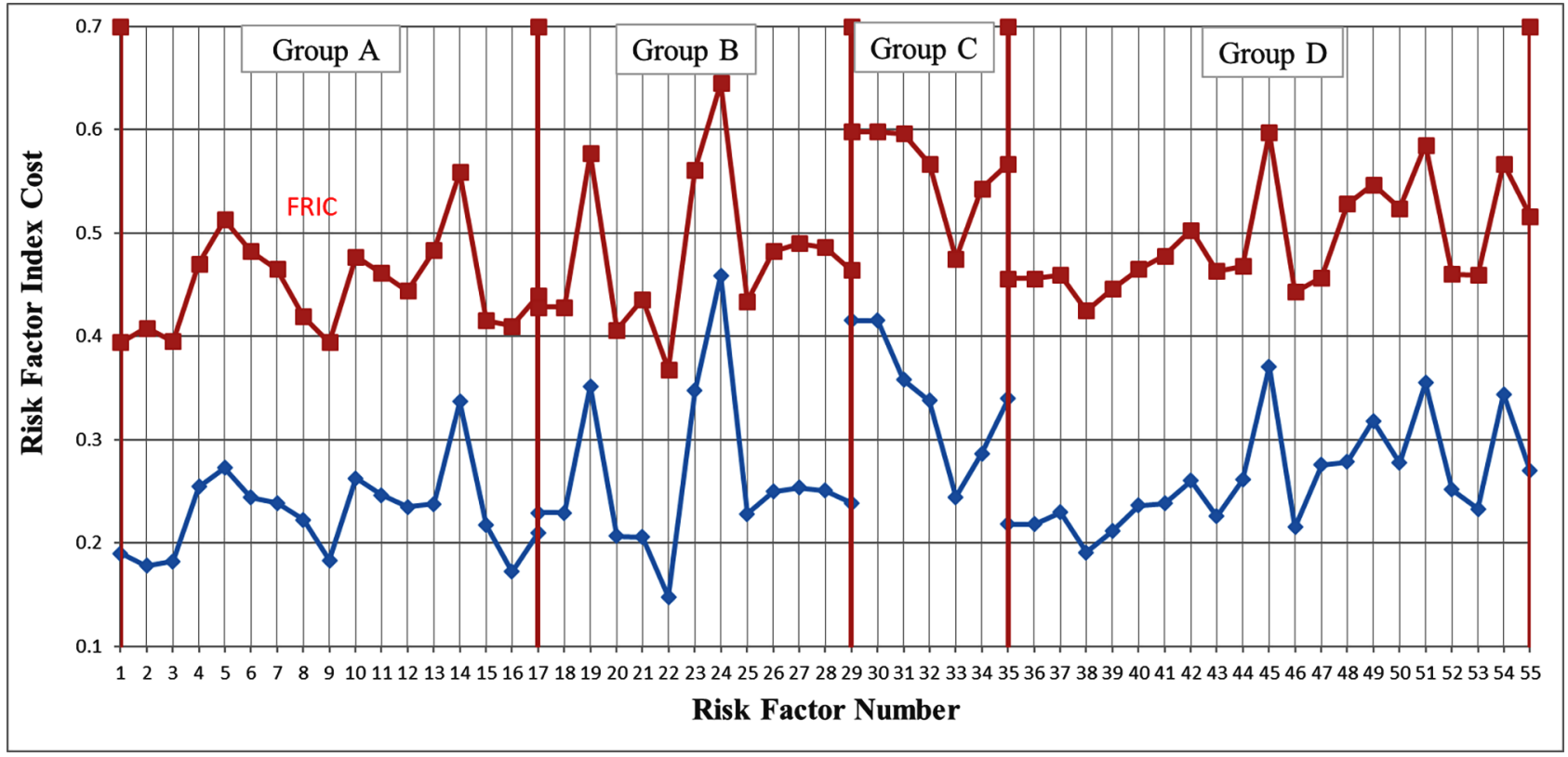

Figure 5. Fuzzy risk factor index for cost (FRIC) and risk factors index for cost (RFIC) 


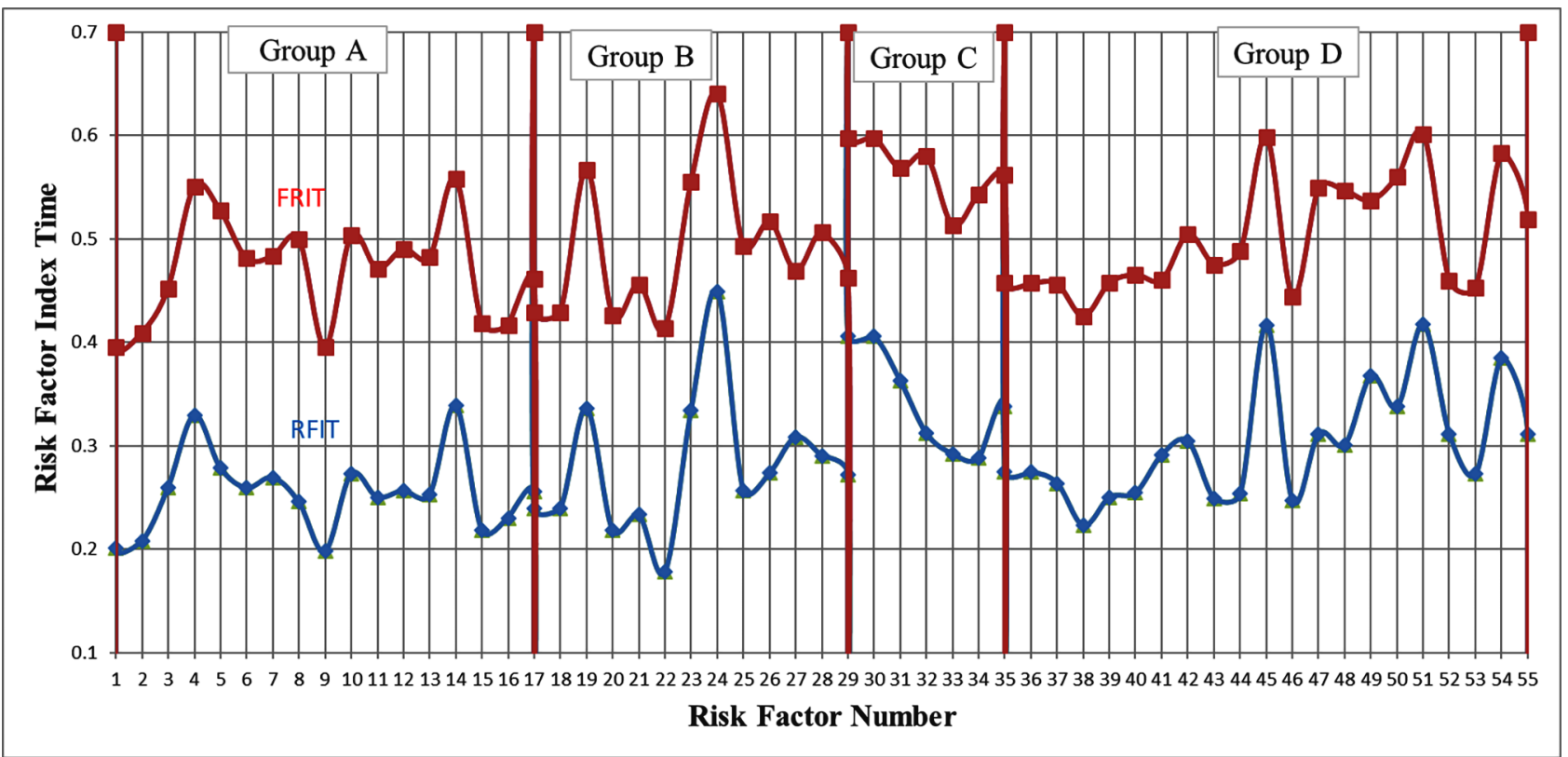

Figure 6. Fuzzy risk factor index for time (FRIT) and risk factors index for time (RFIT)

the outputs are the Fuzzy Activity Cost Overruns (FACO) and the Fuzzy Activity Time Delays (FATD) for the same activities. The relation between the fuzzy activity cost overruns and the risk factor severity with regard to cost can be expressed in Eqn (5). The relation between fuzzy activity time delays and risk factor severity with regard to time can be expressed in Eqn (6):

$$
\begin{aligned}
& F A C O=R S_{1}+R S_{2}+R S_{3}+\ldots R S_{n} ; \\
& F A T D=R S_{1}+R S_{2}+R S_{3}+\ldots R S_{n},
\end{aligned}
$$

where FACO and FATD are the cost overruns and time delay values for each activity, $R S$ is the risk severity for cost and time, and $1,2,3, \ldots, n$ is the number of risk factors.

$R S$ is measured based on two risk variables, namely, the probability index $(P I)$ and the impact index for cost and time (IIC and IIT). The relationship among RS, PI, IIC and IIT can be stated in Eqns (7) and (8):

$$
\begin{aligned}
& R S_{i}=\int\left(P I_{i}, I I C_{i}\right) ; \\
& R S_{i}=\int\left(P I_{i}, I I T_{i}\right),
\end{aligned}
$$

where RS is the risk severity, $f$ is the function, $P I$ is the probability index, IIC is the impact index for cost, IIT is the impact index for time, and $i$ is the number of risk factors.

This research introduced two methods to quantify and determine the accurate cost overruns and time delays for the construction project activities, as shown in Figure 7. In the first method, the $C O Q_{1}$ and $T D Q_{1}$ are quantified by Eqns (9) and (10), respectively, after they are determined by the modules FACO and FATD for each activity. Therefore, $C O Q_{1}$ and $T D Q_{1}$ can also be stated in Eqns (9) and (10):

$$
C O Q_{1}=\partial_{\mathrm{A}} F A C O_{\mathrm{A}}+\partial_{B} F A C O_{B}+\partial_{C} F A C O_{C} ;
$$

$$
T D Q_{1}=\partial_{\mathrm{A}} F A T D_{\mathrm{A}}+\partial_{B} F A T D_{B}+\partial_{C} F A T D_{C},
$$

where $C O Q_{1}$ and $T D Q_{1}$ are the cost overruns quantification and time delays quantification, respectively, from the first method; FACO and FATD are fuzzy activity cost overruns and time delays, respectively; is the activity weight for cost and time in the construction projects; and $A, B$, and $C$ are three activities.

In the second method, a new module is created, and the input variables are FACO and FATD for each activity; however, the module output variables are $C O Q_{2}$ and $T D Q_{2}$, as shown in Figure 7. The second step is defining the membership functions. Before the rules can be evaluated, the inputs must be fuzzified according to each of these linguistic sets. The output of the fuzzy risk assessment procedure is the cost overruns risk percent and the time delays percent. These percentages are calculated by using a scale of $1-40 \%$, which represents the maximum percent that can considered when designing the contract (Issa, 2012a). The states of the linguistic variables are defined as follows: VL, $\mathrm{L}, \mathrm{M}, \mathrm{H}$ and $\mathrm{VH}$ to suit the data collected in previous research (Mosaad et al., 2018). The universe of the discourse scale had also chosen using the predefined membership functions to represent the inputs of the model (0 to 1$)$ for the impact indices of the risk factors, whereas ( 0 to 0.4 ) are chosen for the output to represent the expected cost overruns and time delays. Figures 8 and 9 represent the membership functions for the cost overruns quantification and time delays quantification (COQ and $T D Q$ ) inputs and outputs. In this research, the model will be applied to the three activities $(A, B$, and $C$ ) of the HVAC systems (Mosaad et al., 2018). 


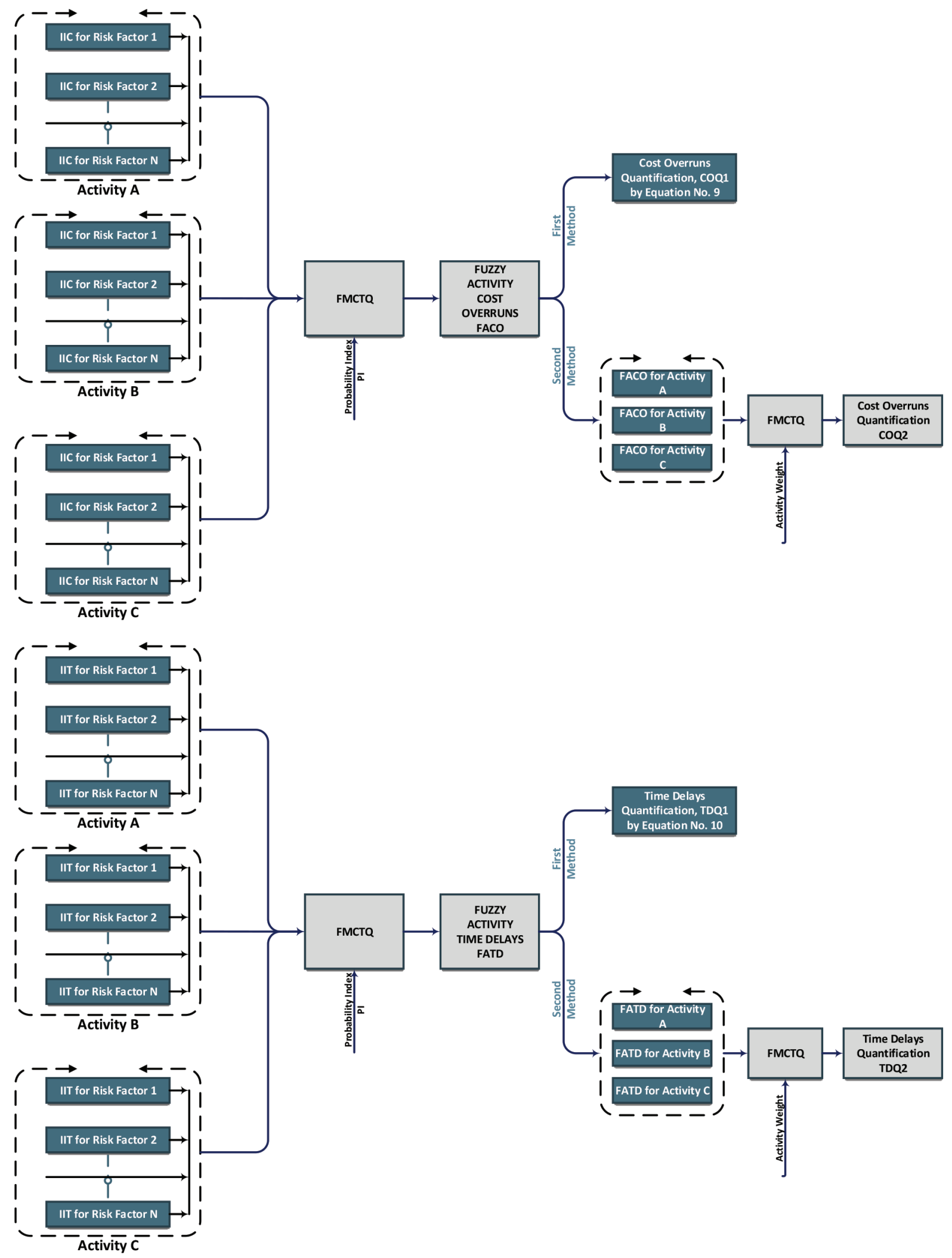

Figure 7. Fuzzy module for cost overruns and time delays quantification (FMCTQ) 


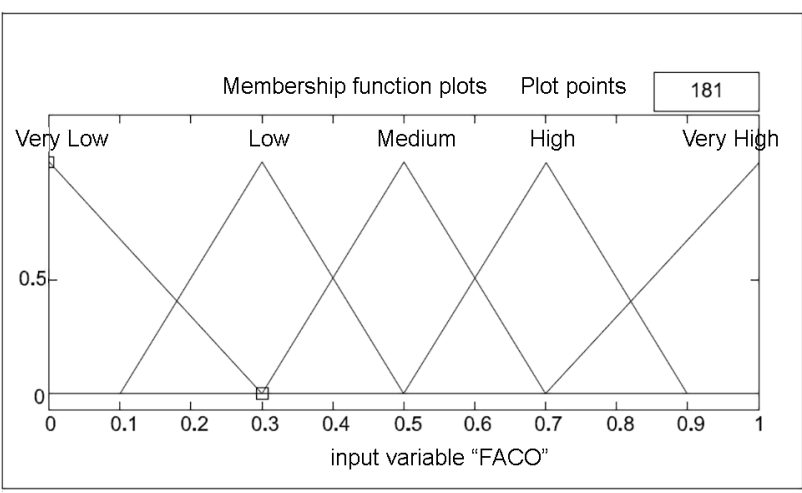

Figure 8. Input membership functions for FACO

\subsubsection{Aggregation and defuzzification process for FMCTQ}

The fuzzy rule base is the basis of the composition or reasoning process of the fuzzy system model. In general, a fuzzy rule base is represented using IF (antecedent) THEN (consequent) (Abd El Khalek et al., 2016). The rule represents the relationship between the input variables and the output variables in terms of linguistic variables instead of mathematical formulas. The rule of the importance index is used when the user does not want the firing of all rules to have equal impact on the output (Issa, 2012a). The probability of the occurrence index for all fifty-five risk factors is calculated and tabulated in order of descending PI (Mosaad et al., 2018). Although all factors may be responsible for the cost overruns and time delays, simultaneously handling all factors is difficult. To resolve this problem, the number of risk factors that affect each activity was identified and reduced to simplify the module application purpose. The number of risk factors were subjectively reduced based on the level of the probability index. Given that 10 input variables in the module and 5 linguistic terms represent each of the variables, the number of If-then rules should be 50 for each activity. Only the 10 top- ranked factors for each activity will be considered for further analysis. Handling 10 factors is possible because they are the factors that have the greatest effect on cost overruns and time delays for each activity. The module has quantified the fuzzy activity cost overruns and time delay values for each activity $(A, B$ and $C)$. Eqns (9) and (10) are used to estimate the $C O Q_{1}$ and $T D Q_{1}$ for the first method. A new module will be created for the second method as follows.

This relation can be represented by a triple premise rule:

- If fuzzy activity cost overruns for activity $A$ and fuzzy activity cost overruns for activity $B$ and fuzzy activity cost overruns for activity $C$, then cost overruns quantification;

- If fuzzy activity time delays for activity $A$ and fuzzy activity time delays for activity $B$ and fuzzy activity time delays for activity $C$, then time delays quantification.

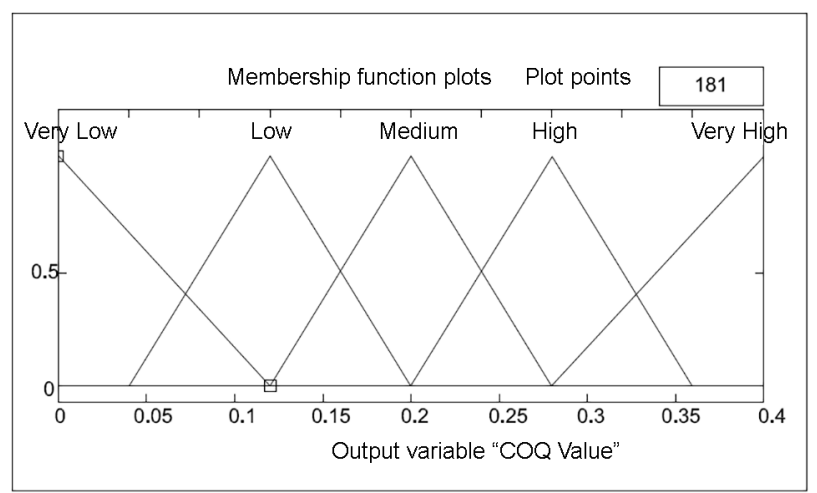

Figure 9. Output membership functions for $\mathrm{COQ}_{2}$

The inputs $\left(F A C O_{A}, F A C O_{B}\right.$ and $\left.F A C O_{C}\right)$ lead to the output $\left(C O Q_{2}\right)$. The inputs $\left(F A T D_{A}, F A T D_{B}\right.$ and $\left.F A T D_{C}\right)$ lead to the outputs $\left(T D Q_{2}\right)$.

Mathematically, the rule can be transformed to the following rule:

If $\left(F A C O_{A}\right)$ and $\left(F A C O_{B}\right)$ and $\left(F A C O_{C}\right)$, then $\left(\mathrm{COQ}_{2}\right)$.

- If fuzzy activity time delays for activity $A$ and fuzzy activity time delays for activity $B$ and fuzzy activity time delays for activity $C$, then time delays quantification;

- If $\left(F A T D_{A}\right)$ and $\left(F A T D_{B}\right)$ and $\left(F A T D_{C}\right)$ then $\left(T D Q_{2}\right)$.

The rules can be readily represented by the matrix shown in Table 2.

A sample of rules extracted from the FAMs matrix for the second method are listed as follows:

- If the $F A T D_{A}$ is very low and the $F A T D_{B}$ is medium and the $F A T D_{C}$ is very low, then the $T D Q_{2}$ is low;

- If the $F A T D_{A}$ is high and the $F A T D_{B}$ is medium and the $F A T D_{C}$ is low, then the $T D Q_{2}$ is medium;

- If the $F A T D_{A}$ is medium and the $F A T D_{B}$ is high and the $F A T D_{C}$ is medium, then the $T D Q_{2}$ is medium;

- If the $F A T D_{A}$ is very high and the $F A T D_{B}$ is high and the $F A T D_{C}$ is high, then the $T D Q_{2}$ is high.

\subsubsection{Model verification and case study projects}

The purpose of the model verification is to show that the results obtained from the model are realistic. The Jabal Omar Development Project is one of the most important and largest projects in Saudi Arabia in terms of location and budget. This project is a unique urban regeneration scheme that is aimed at providing first class accommodations and supporting religious, social and commercial facilities and services for the visitors and residents of the holy city of Makkah Al-Mukarramah. The development will significantly enhance accommodations and other facilities for the ever-increasing number of pilgrims and seasonal visitors to Al-Haram. The Jabal Omar Development Project is a mixed-use development that includes residential apartments, hotels, commercial uses, public 
Table 2. Second method rules for calculating the output of $C O Q_{2}$ and $T D Q_{2}$

\begin{tabular}{|c|c|c|c|c|c|c|c|c|c|c|c|c|c|c|c|c|c|c|c|c|c|c|c|c|c|c|}
\hline \multicolumn{2}{|c|}{ Activity A } & VL & L & M & H & VH & VL & L & M & H & VH & VL & L & M & H & VH & VL & L & M & H & VH & VL & L & M & H & VH \\
\hline & VL & VL & VL & VL & VL & VL & VL & VL & VL & VL & VL & VL & VL & L & L & L & L & L & L & M & M & M & M & M & M & M \\
\hline & L & VL & VL & L & L & L & L & L & L & L & L & L & L & L & L & L & M & M & M & M & M & M & M & M & M & M \\
\hline
\end{tabular}

parking and associated support facilities, such as public assembly and prayer areas. The project encompasses a land area of $230,000 \mathrm{~m}^{2}$ and a built-up floor area of approximately 2.5 million square meters that is intended to be a high-density development (1,500 persons/hectare). The expected construction cost of the Jabal Omar Development is $\$ 5.3$ billion. The second author of this research was a member of one of the project management groups that managed and supervised the construction of the project. The retail and commercial building is the first selected case study project, whereas the residential building is the second case study. The data of the two cases studies are summarized in Tables 3 and 4. The data of the two cases studies are separately fed in the model, and then the model automatically predicts the cost overruns and time delays.

Figure 10 shows the cost overruns quantification percentage for the actual values with the two methods for quantifying the cost overruns. The variance between the quantified cost overruns from the first method compared with the actual values for the two case study projects are
$6.8 \%$ and $4.3 \%$, whereas the variance between the second method and the actual values for the same two case studies are $1.3 \%$ and $1 \%$. Figure 11 shows the time delays quantification percentage and the variance for the quantified time delays for both methods compared with the actual values for the two real cases studies. The results are $8.5 \%$ and $7.4 \%$ for the first method and $1.8 \%$ and $2 \%$ for the second method, which indicates that the model results are too close to the actual projects data and the second method is more accurate than the first method.

\subsection{Model limitations}

There are three major limitations in this study concern the proposed Fuzzy Risk Analysis Model. First, the study focused on three main activities in the construction projects only, so the users should adjust the risk factors under three groups (activities) only. Second, the number of linguistic terms used in model inputs and outputs are limited to five only. In some cases, using more than five linguistic terms, especially in model inputs, may give more accurate results.

Table 3. Project data collected for cost overruns quantification

\begin{tabular}{|c|l|l|c|c|c|c|}
\hline $\begin{array}{c}\text { Phase } \\
\text { No. }\end{array}$ & \multicolumn{1}{|c|}{$\begin{array}{c}\text { Phase } \\
\text { Type }\end{array}$} & $\begin{array}{c}\text { Delivery } \\
\text { Method }\end{array}$ & $\begin{array}{c}\text { Original Cost } \\
\text { \$ Millions) }\end{array}$ & $\begin{array}{c}\text { Actual Cost } \\
\text { Overrun } \\
(\$ \text { Millions })\end{array}$ & $\begin{array}{c}\text { Quantified Cost } \\
\text { Overruns by First } \\
\text { Method (\$ Millions) }\end{array}$ & $\begin{array}{c}\text { Quantified Cost } \\
\text { Overrun by Second } \\
\text { Method (\$ Millions) }\end{array}$ \\
\hline 1 & $\begin{array}{l}\text { Retail, } \\
\text { Commercial }\end{array}$ & Design-Build & 12.00 & 3.84 & 3.02 & 3.72 \\
\hline 2 & Residential & Design-Bid-Build & 6.04 & 1.56 & 1.30 & 1.48 \\
\hline
\end{tabular}

Table 4. Project data collected for time delays quantification

\begin{tabular}{|c|l|l|c|c|c|c|}
\hline $\begin{array}{c}\text { Phase } \\
\text { No. }\end{array}$ & \multicolumn{1}{|c|}{$\begin{array}{c}\text { Phase } \\
\text { Type }\end{array}$} & $\begin{array}{c}\text { Delivery } \\
\text { Method }\end{array}$ & $\begin{array}{c}\text { Original } \\
\text { Duration } \\
\text { (Months) }\end{array}$ & $\begin{array}{c}\text { Actual Time Delay } \\
\text { (Months) }\end{array}$ & $\begin{array}{c}\text { Quantified Time } \\
\text { Delays by First } \\
\text { Method (Months) }\end{array}$ & $\begin{array}{c}\text { Quantified Time } \\
\text { Delays by Second } \\
\text { Method (Months) }\end{array}$ \\
\hline 1 & $\begin{array}{l}\text { Retail, } \\
\text { Commercial }\end{array}$ & Design-Build & 18 & 6.0 & 4.5 & 5.7 \\
\hline 2 & Residential & Design-Bid-Build & 16 & 4.5 & 4.1 & 4.2 \\
\hline
\end{tabular}




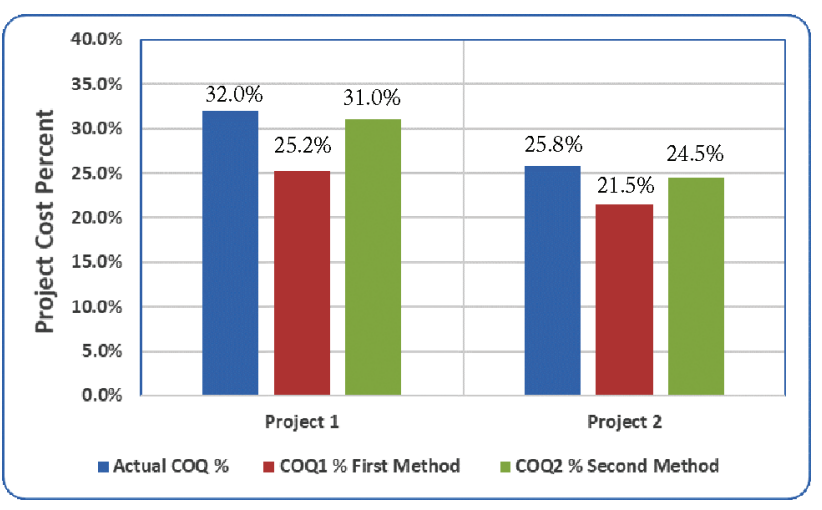

Figure 10. Cost overruns quantification percent

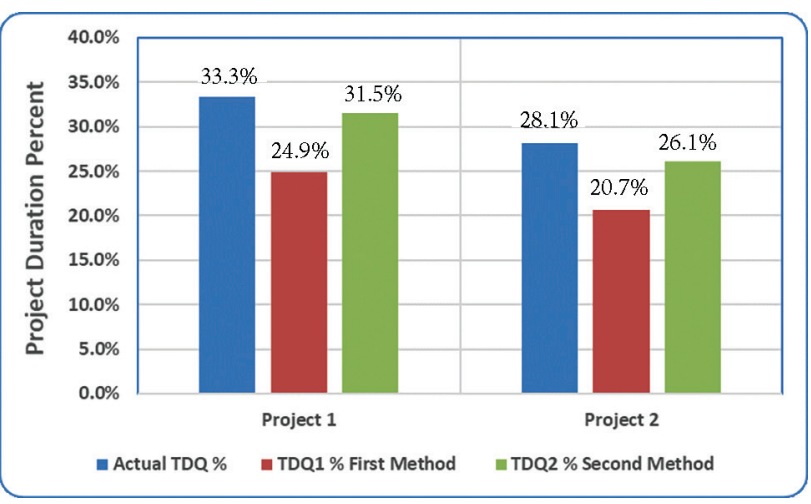

Figure 11. Time delays quantification

Finally, the third limitation concerns inputs range based on fuzzy IF-Then rules. For example, minimum value for input is 0.1 , not zero. This means that no selection chance for case of zero risks. However, these limitations can be addressed in future researches.

\section{Conclusions}

This study introduces a new risk analysis model that is based on a fuzzy logic tool to assess the risks and quantify cost overruns and time delays associated with construction project activities. The model is developed by creating two different modules that address the main activities of construction projects and the associated risks. The first module is developed to evaluate and prioritize the risk factors from combining the probability of the occurrences and impacts on cost or time of activities. The second module is developed to quantify the cost overruns and time delays that are based on many relationships that concern the impacts of the risk factors on activities time and cost by weighting many logical rules. The cost and time of an HVAC system installation represent significant percentages of the total cost and time of construction projects; thus, it has been selected as a case study. The identified HVAC system main activities in this study are $(A)$ air distribution duct work, $(B)$ chilled water distribution pipe work, and (C) HVAC equipment installation. Fifty-five risk factor characteristics that affect these activities and common risk factor characteristics are employed to feed the model. The quantitative approach module has calculated the expected cost overruns and time delays based on the two methods. The first method uses 25 logical rules for each activity, while the second method handles 125 rules for combining the three activities. The model has been tested and verified by an application on two real case study projects in Saudi Arabia using HVAC system activities. A summary of the specific conclusions from this study are as follows:

1. The Spearman and Kendall correlation coefficients were used to confirm the best choice of membership function of four different types. The triangular membership function type is selected as the most suitable function for the proposed model followed by the Gaussian function.

2. The results of assessing the risk factors that affect HVAC activities showed that the most important risk factor that caused the cost overruns and time delays of activities $(A),(B)$, and $(C)$ are "wrong selection of dampers and plenum boxes", "chilled water distribution pipe work is poor selection of valves that might cause more damage" and "wrong selections that might cause future rectification", respectively.

3. The maximum variance between the quantified cost overruns and the time delays from the triple premise rule compared with actual values for the two case study projects are $1.3 \%$ and $2 \%$, respectively. The quantified values from the first method are $6.8 \%$ and $8.5 \%$ for the cost overruns and time delays, respectively.

4. It is recommended that project managers that work with HVAC systems should consider the major risk factors presented in this study, which affect the activities and caused cost overruns and time delays.

5. The presented model is not limited to HVAC system activities but can be applied to all construction project activities.

\section{Funding}

No fund for this article.

\section{Disclosure statement}

None of the authors of this article has a financial or personal relationship with other people or organizations that could inappropriately influence or bias the content of the article. It is to specifically state that "No Competing interests are at stake and there is No Conflict of Interest" with other people or organizations that could inappropriately influence or bias the content of the article.

\section{References}

Abd El Khalek, H., Aziz, R. F., \& Kamel, H. (2017). International construction projects' risk cost estimation: Fuzzy logic and AHP in application (Real case studies). Journal of Buildings and Sustainability, 2(2). 
Abd El Khalek, H., Aziz, R. F., \& Kamel, H. M. (2016). Risk and uncertainty assessment model in construction projects using fuzzy logic. American Journal of Civil Engineering, 4(1), 24-39. https://doi.org/10.11648/j.ajce.20160401.13

Ahmed, S. A., Issa, U. H., Farag, M. A., \& Abdelhafez, L. M. (2013). Evaluation of risk factors affecting time and cost of construction projects in Yemen. International Journal of Management, 4(5), 168-178.

Asadi, P., Zeidi, J. R., Mojibi, T., Yazdani-Chamzini, A. Y., \& Tamosaitiene, J. (2018). Project risk evaluation by using a new fuzzy model based on ELENA guideline. Journal of Civil Engineering and Management, 24(4), 284-300. https://doi.org/10.3846/jcem.2018.3070

Carr, V., \& Tah, J. H. M. (2000). A proposal for construction project risk assessment using fuzzy logic. Construction Management and Economics, 18, 491-500. https://doi.org/10.1080/01446190050024905

Carr, V., \& Tah, J. H. M. (2001) A fuzzy approach to construction project risk assessment and analysis construction project risk management system. Advances in Engineering Software, 32, 847-857. https://doi.org/10.1016/S0965-9978(01)00036-9

Cheng, J., Xu, S.-M., \& Chen, Z.-R. (2018, August). A fuzzy logic-based method for risk assessment of bridges during construction. In The 2018 Structures Congress (Structures 18) (pp. 27-31). Songdo Convensia, Incheon, Korea.

Dikmen, I., Birgonul, M. T., \& Han, S. (2007). Using fuzzy risk assessment to rate cost overrun risk in international construction projects. International Journal of Project Management, 25, 494-505. https://doi.org/10.1016/j.ijproman.2006.12.002

Gohar, A. S., Khanzadi, M., \& Farmani, M. (2012). Identifying and evaluating risks of construction projects in fuzzy environment: A case study in Iranian construction industry. Indian Journal of Science and Technology, 5, 3593-3602.

Idrus, A., Nuruddin, M. F., \& Rohman, M. A. (2011). Development of project cost contingency estimation model using risk analysis and fuzzy expert system. Expert Systems with Applications, 38, 1501-1508. https://doi.org/10.1016/j.eswa.2010.07.061

Issa, U. H. (2012a). A model for time overrun quantification in construction of industrial projects based on risk evaluation. Journal of American Science, 8(8), 523-529.

Issa, U. H. (2012b). Developing an assessment model for factors affecting the quality in the construction industry. Journal of Civil Engineering and Architecture, 6(3), 364-371. https://doi.org/10.17265/1934-7359/2012.03.010

Issa, U. H., \& Ahmed, A. (2014). On the quality of driven piles construction based on risk analysis. International Journal of Civil Engineering, Transaction B: Geotechnical Engineering, 12.

Issa, U. H., \& Salama, I. M. (2018). Improving productivity in Saudi Arabian construction projects: An analysis based on Lean techniques. International Journal of Applied Engineering Research, 13(10), 8669-8678.

Issa, U. H., Farag, M. A., Abdelhafez, L. M., \& Ahmed, S. A. (2015). A risk allocation model for construction projects in Yemen. Civil and Environmental Research, 7(3).
Issa, U. H., Miky, Y. H., \& Abdel-Malak, F. F. (2019). A decision support model for civil engineering projects based on multi-criteria and various data. Journal of Civil Engineering and Management, 25(2), 100-113.

https://doi.org/10.3846/jcem.2019.7551

Khazaeni, G., Khanzadi, M., \& Afshar, A. (2012). Optimum risk allocation model for construction contracts: fuzzy TOPSIS approach. Canadian Journal of Civil Engineering, 39(7), 789800. https://doi.org/10.1139/12012-038

Kosko, B. (1992). Neural networks and fuzzy systems. PrenticeHall.

Morote, A. N., \& Vila, F. R. (2011). A fuzzy approach to construction project risk assessment. International Journal of Project Management, 29, 220-231.

https://doi.org/10.1016/j.ijproman.2010.02.002

Mosaad, S. A. A., Issa, U. H., \& Hassan, M. S. (2018). Risks affecting the delivery of HVAC systems: Identifying and analysis. Journal of Building Engineering, 16, 20-30. https://doi.org/10.1016/j.jobe.2017.12.004

Nawar, S. E. M., Hosny, K., \& Nassar, K. (2017). Owner time and cost contingency estimation for building construction projects in Egypt (Master's thesis). School of Sciences and Engineering, the American University in Cairo. https://doi.org/10.1061/9780784481271.036

PM Institute. (2004). A guide to the project management body of knowledge (PMBOK Guide) ( $3^{\text {rd }}$ ed.). USA.

Rezakhani, P. (2011). Fuzzy risk analysis model for construction projects. International Journal of Civil and Structural Engineering, 2(2), 507-522.

Rezakhani, P. (2012). Fuzzy multi criteria decision making model for risk factor selection in construction projects. Buletinul Institutului Polotenic Din IAŞI, 128-142.

Senouci, A., Ismail, A. A., \& Eldin, N. (2016). Time and cost overrun in public construction projects in Qatar. In Creative Construction Conference 2016 (pp. 231-236). Budapest, Hungary. https://doi.org/10.1016/j.proeng.2016.11.632

Sharma, S., \& Goyal, P. K. (2014). Cost overrun assessment model in fuzzy environment. American Journal of Engineering Research, 3, 44-53.

Singh, S., \& Trivedi, M. K. (2012). Application of fuzzy logic in delay analysis in construction. International Journal of Computational Engineering Research, 2(2), 599-605.

Yazdani-Chamzini, Y. A. (2014). Proposing a new methodology based on fuzzy logic for tunnelling risk assessment. Journal of Civil Engineering and Management, 20(1), 82-94.

https://doi.org/10.3846/13923730.2013.843583

Zeng, J., An, M., \& Smith, N. J. (2007). Application of a fuzzy based decision-making methodology to construction project risk assessment. International Journal of Project Management, 25, 589-600. https://doi.org/10.1016/j.ijproman.2007.02.006

Zhang, G., \& Zou, P. X. W. (2007). Fuzzy analytical hierarchy process risk assessment approach for joint venture construction projects in China. Journal of Construction Engineering and Management, 133, 771-779.

https://doi.org/10.1061/(ASCE)0733-9364(2007)133:10(771) 